
\title{
Independent Object Tracking from Video using the Contour Information in HSV Color Space
}

\author{
Jong Hun Park', Gang Seong Lee ${ }^{2 *}$, Jin Soo Kim³ ${ }^{3}$ Seuc Ho Ryu ${ }^{4}$ and Sang Hun Lee \\ 'Department of Plasmadiodisplay, Kwangwoon University, Seoul, 01897, Korea; qwerty@kw.ac.kr \\ 2Ingenium College of Liberal Arts, Kwangwoon University, Seoul, 01897, Korea; gslee@kw.ac.kr, leesh58@kw.ac.kr \\ ${ }^{3}$ IDP System, Co. Ltd, Seoul, 07646, Korea \\ ${ }^{4}$ Deptartment of Game Design, Kong Ju National University, Kong Ju, 32588, Korea
}

\begin{abstract}
Background/Objectives: In this paper, a tracking method that is based on the colors and contours is proposed to obtain results that are superior to those of the traditional methods for the tracking of the objects in video. Methods/Statistical Analysis: The proposed method requires an initial conversion of the existing RGB-color-based video in the HSV color space, and a back-projection histogram in the HSV color space is used to obtain the color information of the object region that is to be tracked. The contour information is also obtained to track the target based on the Canny algorithm, whereby the moving object in the video is tracked in real time on the basis of the color and contour information. Findings: An experiment was performed wherein the proposed method was compared with the Mean-shift tracking method, the Camshaft tracking method, the Kalman-filter tracking method, and the Particle-filter tracking method in terms of the objective of this paper. The comparison showed that the proposed method is more accurate regarding the tracking of a single object in video. Improvements/Applications: This improved tracking method will be utilized in object tracking, CCTV for car tracking and HCI.
\end{abstract}

Keywords: Canny Algorithm, Contour Detection, Histogram Back-Projection, Object Tracking, RGB-to-HSV Conversion

\section{Introduction}

Computer vision is one of the research areas that has received attention for the analysis and understanding of the scene or the features of an imagen. Object tracking is one of the computer-vision techniques that tracks the position change of a target through the detection of a specific object in a video. The application of objecttracking technology includes the person-monitoring system, HCI (Human-Computer Interaction), CCTV for car-tracking, and car-number recognition systems, The typical object-tracking methods that have been proposed are the tracking method for which the Mean-shift is used and the tracking method for which the Cam-shaft ${ }^{\text {| }}$ is used. The advantage of the conventional methods is simplicity, whereby the reduced computational complexity requires less implementations so that a faster operation is possible; however, the tracking of a target in a variety of environments such as those wherein the lighting changes, objects move rapidly, or the object size changes is not accurate. For the achievement of correct object tracking in this paper, the color space of the image is first converted from the RGB color space to the HSV color space; then, the destination information and the color of its surroundings is obtained with the use of a histogram that tracks the back-projection and the Canny algorithm for the attainment of the contour information of the target track. It is the tracking of the object that is based on the color information and the contour information of the target that provides results that are more accurate than those of the conventional tracking methods. 


\subsection{RGB-to-HSV Conversion}

In general, an RGB color space will produce a different color in terms of the Red, Green, and Blue after the addition of the three colors? The RGB color space is sensitive to color changes, but it is insusceptible to changes of the intensity characteristic; therefore, it is difficult to correct the object tracking if a video has occurred a large impact from changes of the lighting element and the external environment? To solve this problem, the progress in the HSV color space that includes the $\mathrm{V}$ (Value) of the brightness value of the video is tracked according to the RGB-to-HSV conversion. Equations (1), (2), and (3) illustrate the conditions for RGB to HSV color transformation 9 .

$$
\begin{aligned}
& R^{\prime}=\frac{R}{255}, G^{\prime}=\frac{G}{255}, B^{\prime}=\frac{B}{255} \\
& C_{\text {mas }}=\max \left(R^{\prime}, G^{\prime}, B^{\prime}\right) \\
& C_{\text {min }}=\min \left(R^{\prime}, G^{\prime}, B^{\prime}\right)
\end{aligned}
$$

RGB to HSV conversion for $\mathrm{H}, \mathrm{S}, \mathrm{V}$ is defined by Equations (4), (5), and (6).

$$
\begin{aligned}
& \Delta=C_{\text {max }}-C_{\text {min }} \\
& S= \begin{cases}0 & , C_{\text {mas }}=0 \\
\frac{\Delta}{c_{\text {max }}} & , C_{\text {max }} \neq 0\end{cases} \\
& V=C_{\text {max }}
\end{aligned}
$$

Figure 1 shows the results of applying RGB to HSV transformation to an image.

\subsection{Histogram Back-Projection}

The image-processing histogram comprises the pixel values of the image analysis and is often used in image analyses 10. In this paper, because the histogram detects the changes of the contour and color information between the consecutive frames in the video, it helps in the analysis of the located object pixel ${ }^{11}$. The $\mathrm{x}$-axis that represents the pixel histogram with the values of brightness, contrast, and color is composed of a constant-size bin; alternatively, the $y$-axis indicates the frequency that is contained in the respective bin, whereby the number of pixels is represented. The operation to obtain a histogram in the HSV color space is defined by Equation (7).

$$
h=\sum_{j=0}^{n} r_{i}\left(x_{j}\right)
$$

The histogram back-projection is a method for which a proportion of the value of the image histogram is used to obtain the tracked-histogram value of the target that is to be tracked. To perform a back-projection, the attainment of the three-channel HSV histogram should first occur so that the object can be tracked. Next, the image-histogram values are compared to perform the histogram and the tracking of the object that is to be tracked.

An algorithm for the comparison of the histogram values typically comprises four methods 1 . The Correlation is calculated here so that it can be used by the normal correlation operator to measure the degree of similarity between the two sample values defined by Equation (8).

$$
C\left(h_{1}, h_{2}\right)=\frac{\sum_{i} h_{1}(i) \cdot h_{2}(i)}{\sqrt{\sum_{i} h_{1}^{2}(i)^{2}(i)} \cdot h_{2}^{2}(i)}(8)
$$

where $h_{n}=h_{n}(i)-\frac{1}{N} \sum_{j} h_{n}(j)$ and $N$ is the number of histogram bins. The Intersection is calculated as a way to compare the sum of the two min. values and is defined as Equation (9).

$$
I\left(h_{1}, h_{2}\right)=\sum_{i} \min \left(h_{1}(i), h_{2}(i)\right)
$$

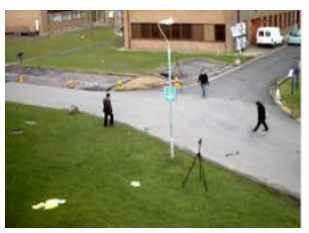

(a)

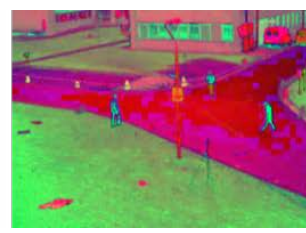

(b)

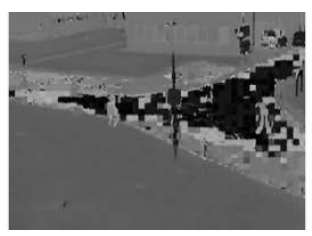

(c)

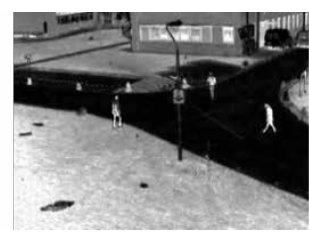

(d)

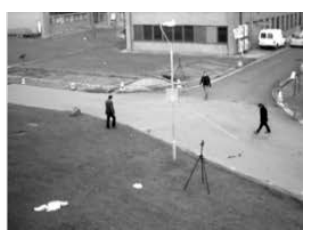

(e)

Figure 1. RGB-to-HSV color space transform. (a) RGB image. (b) HSV image. (c) H-space image. (d) $\mathrm{S}$-space image. (e) V-space image. 


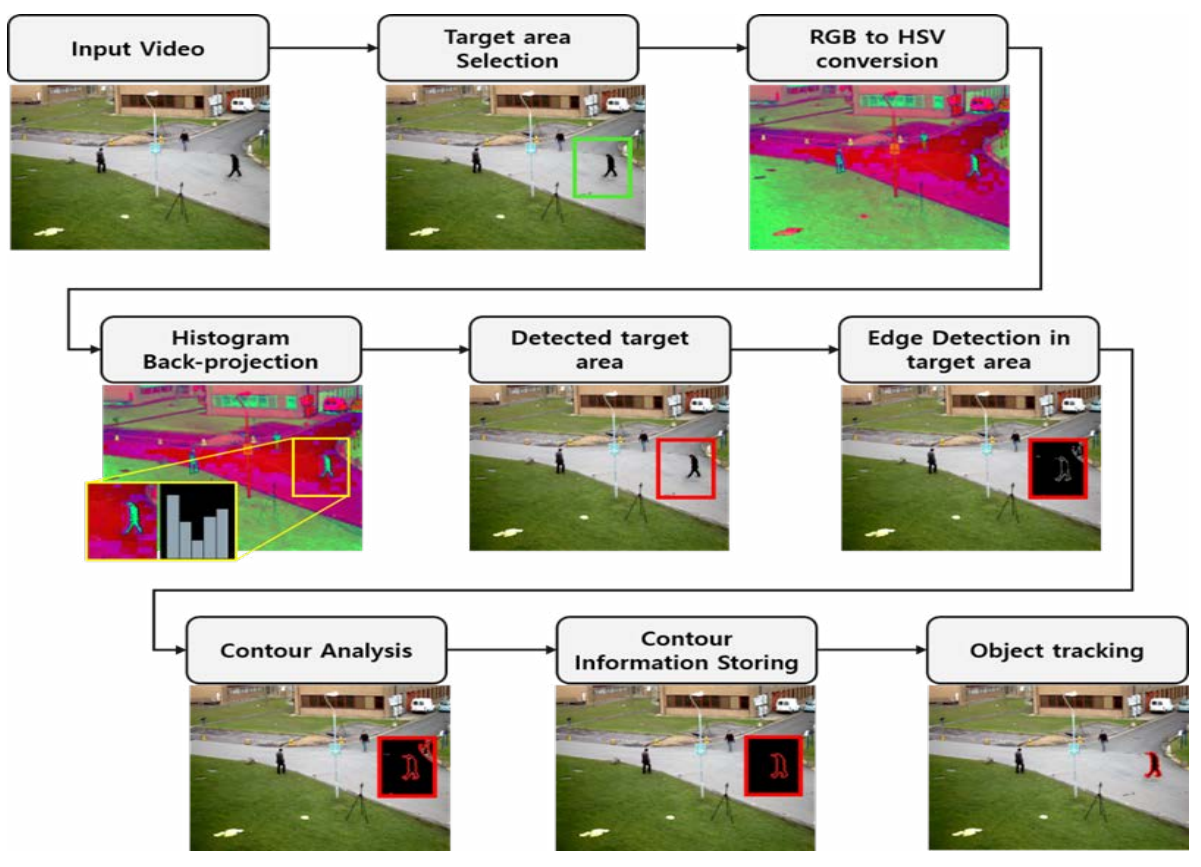

Figure 2. Flow chart of the proposed method.

The Chi-square is a method for the measurement of the degree of similarity whereby the difference of the regular square of each value is used, and the operation for the method is defined as Equation (10).

$C s\left(h_{1}, h_{2}\right)=\sum_{i} \frac{\left(h_{1}(i)-h_{2}(i)\right)^{2}}{h_{1}(i)+h_{2}(i)}$

The Bhattacharyya distance is a statistical method for the measurement of the similarity between two probability distributions; when fully matched, its value is 0 , and a mismatch results in a value of 1 . The closer that the distance is to 0 , the greater the likelihood that the two values are similar.The operation of the Bhattacharyya distance for histogram comparison is defined as Equation (11).

$B\left(h_{1}, h_{2}\right)=\sqrt{1-\sum_{i} \frac{\sqrt{h_{1}(i) \cdot h_{2}(i)}}{\sqrt{\sum_{i} h_{1}(i) \cdot \sum_{i} h_{2}(i)}}}$

Using these four methods, the histogram values of the target are compared with the histogram values of the image to perform the tracking, and the most similar parts of the histogram (the probability of having the highest value of the histogram part) are then found using the object-tracking model in the video frame. Every time each frame is in progress, the repeated performance of this operation tracks the object. In this paper, the histogram back-projection was performed using the Bhattacharyya distance after the histogram comparison.

\section{The Proposed Method}

The flowchart of the proposed method is shown in Figure 2.

Regarding the conventional object-tracking algorithm, for which the approximate range of the target to track is identified, there is a limit that hampers the attainment of accurate object-tracking results; furthermore, it is difficult to determine the color information as only an ambiguous distinction is evident between the background and foreground colors. This algorithm therefore identifies the approximate location, the distribution of the target track is used to identify the histogram back-projection from the video, and the contour information of the target track is used to obtain the accurate object-tracking results. To obtain the edge of the target area, the contour information of the tracking object is based on the Canny algorithm. Then, the major edge pixel that is determined as the object is discriminated from the remainder (garbage) edge pixel that is from the edge image. The major pixel is stored in a buffer according to the defining by the contour pixel, and the remaining pixel values are discarded. For the tracking of an object, the contour-pixel information that is stored in the buffer is used 


\subsection{Contour Analysis}

After the Canny algorithm is used to obtain an edge in the target area, two conditions are used to determine whether each of the edge pixels of the contour pixel of the object are included as part of the tracking process. The first condition is the pixel continuity. The contour of an object consists of a point of the continuous pixel, the non-contiguous parts that are not from a single object, or a greater possibility regarding the noise pixel. Therefore, the edge-pixel consecutive points are preferentially classified as contour pixels that belong to the candidate group. The equation for the classification of the contourpixel candidates is as in Equation (12).

$$
C p=\sum_{i=0}^{n} \sum_{j=0}^{m} C p_{x i}+C p_{y i}+C p_{d i, j}
$$

where $C p_{x i}$ and $C p_{y i}$ are the $\mathrm{x}$-axis and $\mathrm{y}$-axis, respectively, and $C p_{d i, j}$ represents the diagonal contourpixel candidates. $C p_{x i}, C p_{y i}$, and $C p_{d i, j}$ are defined as Equation (13), (14), (15).

$$
\begin{aligned}
& C p_{x i}= \begin{cases}1, & x-2, x-1, x+1, x+2=1 \\
0, & x-2, x-1, x+1, x+2=0\end{cases} \\
& C p_{y i}=\left\{\begin{array}{l}
1, y-2, y-1, y+1, y+2=1 \\
0, y-2, y-1, y+1, y+2=0
\end{array}\right. \\
& C p_{d i, j}= \begin{cases}1, & (x-2, y-2),(x-1, y-1),(x+1, y+1),(x+2, y+2)=1 \\
0, & (x-2, y-2),(x-1, y-1),(x+1, y+1),(x+2, y+2)=0\end{cases}
\end{aligned}
$$

$C p_{x i} C p_{y i}$, and $C p_{d i, j}$ are determined by the contourpixel candidates only when all of the neighboring pixel values are determined as 1 on the basis of the center pixel; therefore, in all of the areas of the detecting edge, the application of Equation (13), Equation (14), and Equation (15) can form a continuous object-area edge.

Regarding these methods for the classification of the continuous-edge pixel, the high-linearity edge pixel is mainly classified; therefore, the direction for the classification of an accurate contour pixel is considered. The portion that comprises the object periphery is configured into the most \pm 45 -degree direction.
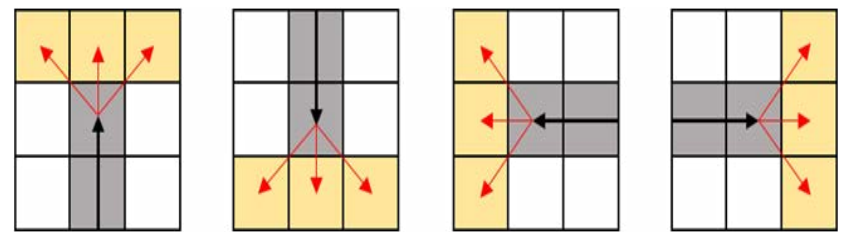

Figure 3. Classification of the continuous-edge pixel for horizontal and vertical directions.
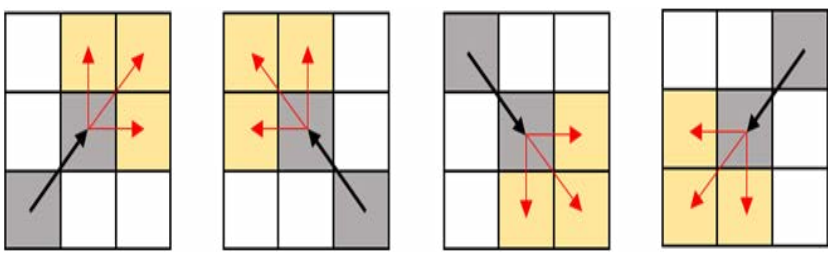

Figure 4. Classification of the continuous-edge pixel for the diagonal direction.

Figure 3 and Figure 4 are schematic diagrams for determining the contour pixel in the horizontal direction, the vertical direction and the diagonal direction. The gray color box is the current contour pixel, the yellow box indicates the directions that belong to a contour pixel. If all of the yellow boxes contain edge pixels, the middle yellow box will be classified as the candidate contour pixel with the lowest direction. However, if the middle yellow box does not contain the edge pixel, the left and right yellow boxes are considered.

Although the classification of the contour-pixel candidates was completed, the formation of a line segment resulted in successive points, and this shows that it is difficult to form a complete contour of the object that is to be tracked. The part has therefore not been detected as an edge pixel within the contour portion that is estimated on the basis of the continuity of the pixel, and it will ultimately form the complete contour of the tracked object. Figure 5 is a contour pixel candidate obtained by considering the continuity and orientation of the pixel.

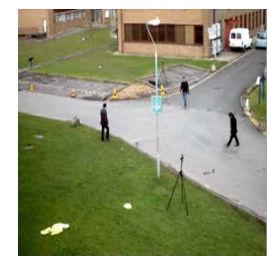

(a)

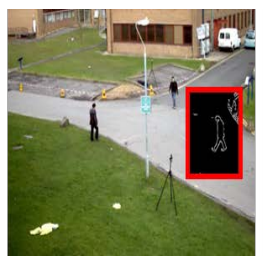

(b)

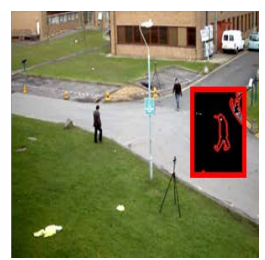

(c)
Figure 5. Canny edge detection and candidate for the contour pixel. (a) Original image. (b) Canny edge detection. (c) Candidate for the contour pixel.

\subsection{Contour Information Storage}

The pixels that belong to the contour of the candidate group were formed after the determination of the edge pixels. But all of the candidates are not the target of the contour pixel that is to be tracked; therefore, by storing only the contour pixel of the destination track in the buffer, the stored pixel should be applied to the frame for the tracking of the comparison group. 
First, the shape of the line for the sighting of the beginning and the end of the pixel candidates shall be excluded. The candidate of a line shape is not the tracked object that most likely belongs to the foreground area as environmental boundaries such as roads, tables, and buildings. And with the exception of the line-shape candidates, the irregular shapes among the candidates, which are close to the center of the target area and are defined by a set of the contour pixels of the target to track the most candidates, are applied to the comparison-group frame. The remaining candidates are removed from the buffer to avoid the slowness problem of real-time object tracking.

Figure 6 is the object-tracking result images for which the outline information of the target track that is stored in the buffer is used are as follows.
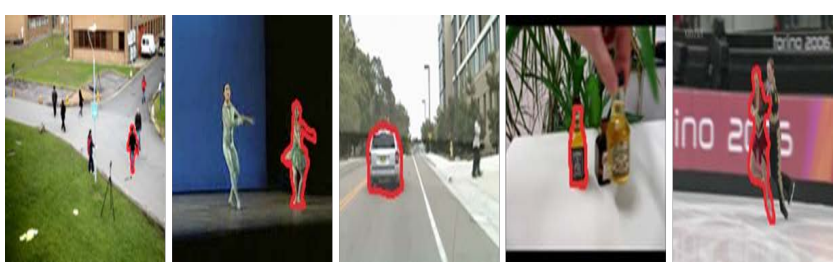

Figure 6. Result images of object tracking.

\section{Experiment and Discussion}

This experiment was developed in Windows 10/Intel core i5-6400 2.70 GHz/4 GB RAM/Visual Studio 2013. For the comparison with the conventional tracking algorithms, the video experiments were performed within a variety of environments. The videos that were used in the experiments were collected from Websites including Google and YouTube. In addition, the results of the experiments with the Cam-shift algorithm, Mean-shift algorithm, Kalmanfilter tracking algorithm 1 , and Particle-filter tracking algorithm ${ }^{\text {m }}$ that are used for the comparison were obtained in environments that are the same as those that were used to obtain the results of the proposed algorithm.

Figure 7 shows the results from tracking a ballerina in a ballet video. The results of tracking the ballerina using Cam-shift showed that although, both the ballerina and the background contained similar colors like blue, the tracking was relatively accurate and the ballerina was placed in the center of the tracking area. However, when the ballerina is in front of a dark background, the frame with the ballerino is inaccurate. The Meanshift showed relatively accurate tracking results for the ballerina. However, the ballerino appeared in the video, and no identification object was tacked after tracking with ballerino. The method using the Kalman filter did not track the ballerina accurately, and the tracking target was missed because tracking was interrupted. The method using the Particle filter was more accurate than the previous three methods, and was applied without missing the tracking target. However, when the objects are densely packed as in the second, fourth, and the fifth images, the results showed only the identification object was not accurately tracked. Finally, the proposed method did not accurately track the ballerina in the second image, but the fifth image shows exact tracking and identification of the Ballerina unlike existing methods.

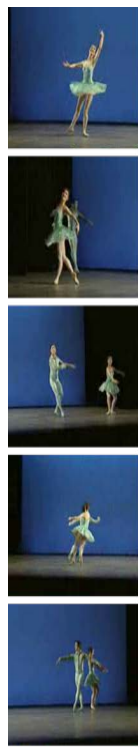

(a)

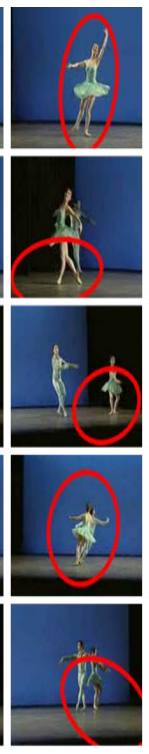

(b)

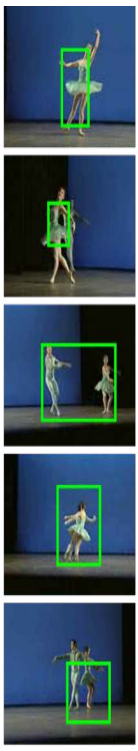

(c)

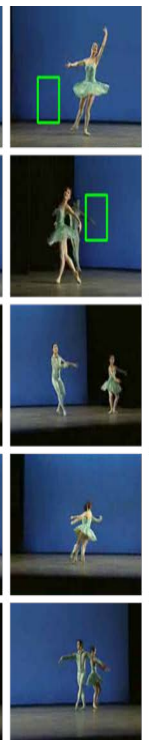

(d)

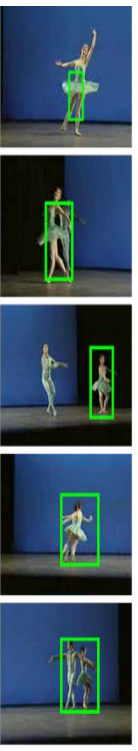

(e)

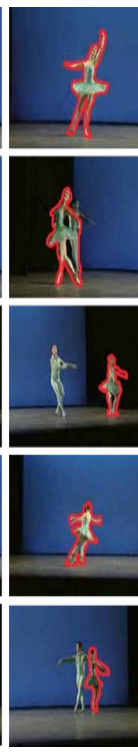

(f)
Figure 7. Ballet video. (a) Original frame. (b) Camshift tracking. (c) Mean-shift tracking. (d) Kalman-filter tracking. (e) Particle-filter tracking. (f) Proposed method.

Figure 8 is an experimental result for tracking a moving car on the road. The method using Cam-shift did not accurately track the car from the beginning of the track, and the final results showed that the trees were tracked. The method using Mean-shift accurately tracked the car but the background around the car was gradually included in the tracking area and resulted in incorrect tracking. The method using the Kalman-filter alternated between tracking the car and the trees. The method using the Particle-filter accurately tracked the car, but the tracking results showed that only a portion of the car. The proposed method shows accurate tracking results following the outline of the car. 


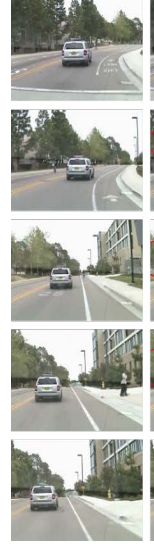

(a)

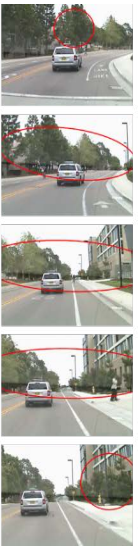

(b)

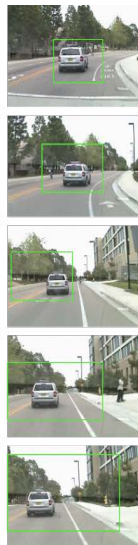

(c)

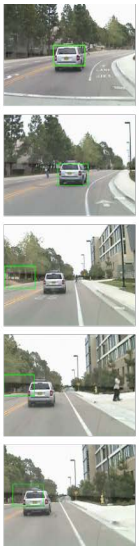

(d)

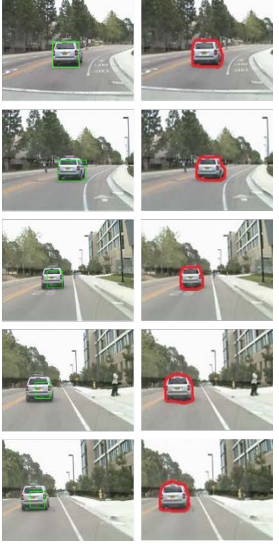

(e) (f)

Figure 8. Car video. (a) Original frame. (b) Cam-shift tracking. (c) Mean-shift tracking. (d) Kalman-filter tracking. (e) Particle-filter tracking. (f) Proposed method.

Figure 9 is shows the results of tracking a bottle on the table. The methods using Cam-shift and Meanshift tracked a different bottle with color similar to the intended item or tracked multiple bottles at the same time. The tracking method using a Kalman-filter accurately tracked the bottle, but missed the target when the object overlapped an object (obstruction) with a similar color. However, re-tracking succeeds and the target is tracked accurately. The method using the Particle filter shows results that accurately track the target until the end of the video. The proposed method shows the results of tracking several objects similar to the Kalman-filter in the fourth image. This results in a significantly higher tracking result than the proposed method using a Particle filter.

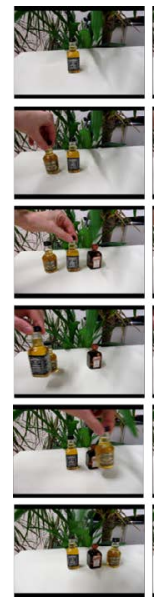

(a)

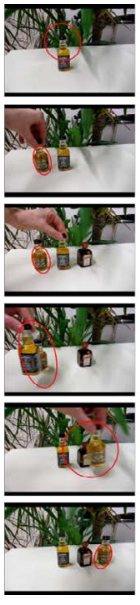

(b)

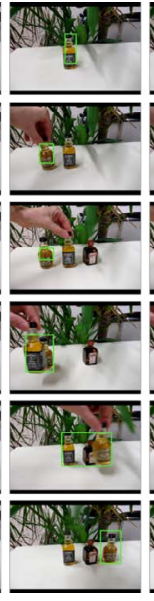

(c)

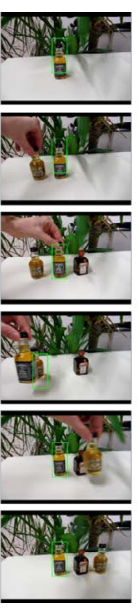

(d)

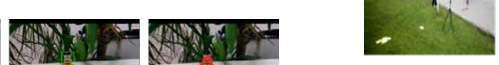

(a)

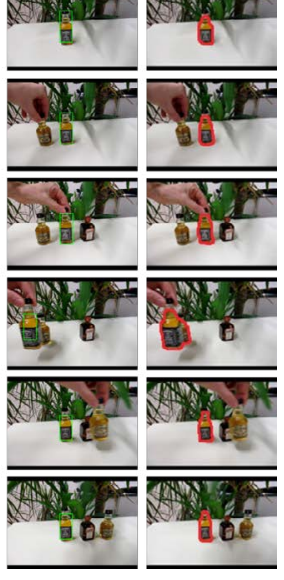

(e) (f)

Figure 9. Bottles video. (a) Original frame. (b) Cam-shift tracking. (c) Mean-shift tracking. (d) Kalman-filter tracking. (e) Particle-filter tracking. (f) Proposed method.

Figure 10 shows the results for tracking one person among multiple people in motion. The Cam-shift method tracked the identification object relatively accurately, but misses the target as the target passed the sign in the middle of the image or overlapped with other moving people. It also seems that the man dressed in similar clothes was also tracked in the fifth image. Mean-shift shows experimental results similar to Cam-shift. In contrast, the method using the Kalman-filter showed precise tracking of the object, but the target was missed in the fourth image where it is overlapped with signs. The method using the Particle-filter had problems with tracking other objects in the fourth image, and retracing the results showed that an identification object was tracked. The proposed method missed the target in the third image but showed accurately tracking of the identification object overall in the other images.

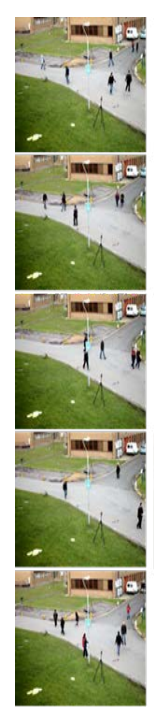

\section{(a)}

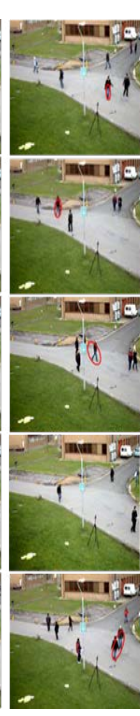

(b)

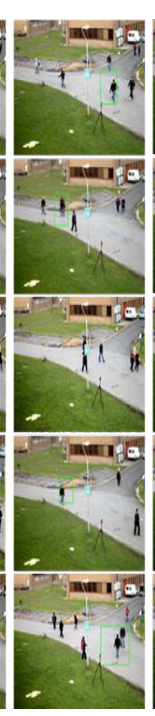

(c)

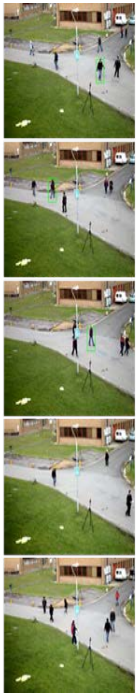

(d)

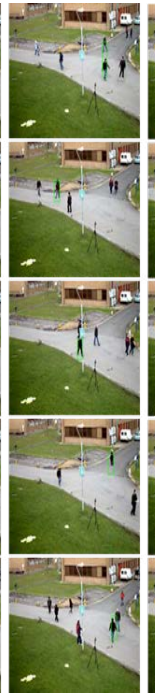

(e)

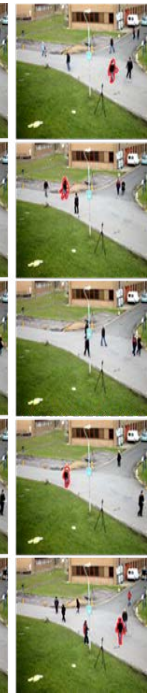

(f)
Figure 10. Bottles video. (a) Original frame. (b) Cam-shift tracking. (c) Mean-shift tracking. (d) Kalman-filter tracking. (e) Particle-filter tracking. (f) Proposed method.

Figure 11 shows the results for a figure skating video, in which a female figure skater was tracked. Cam-shift and Mean-shift methods included two figure skaters tracking in the range, and the results were inaccurate because much of the background was also included. The method using the Kalman-filter tracks the female figure skater, but the tracking results are incorrect when the skater is before a background with a similar color. The Particle-filter tracks only the female figure skater but the results showed that 
some of the background is included. Finally, the proposed method has unstable results showing tracking of the female figure player independently or tracking both figure skaters. However, the results seem to accurately track only the objects without including the background.

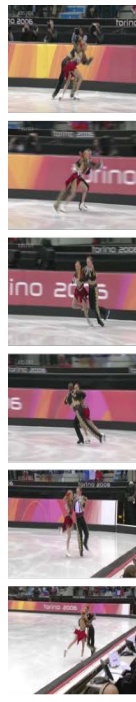

(a)

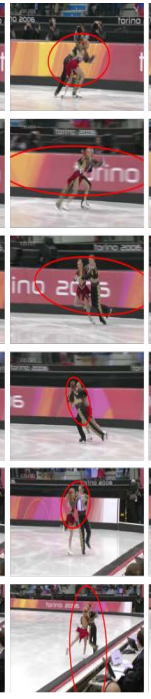

(b)

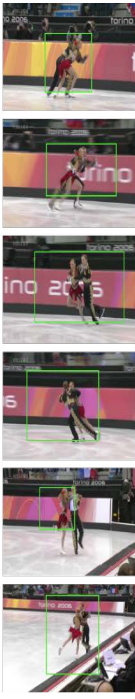

(c)
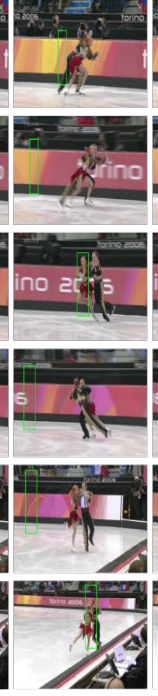

(d)

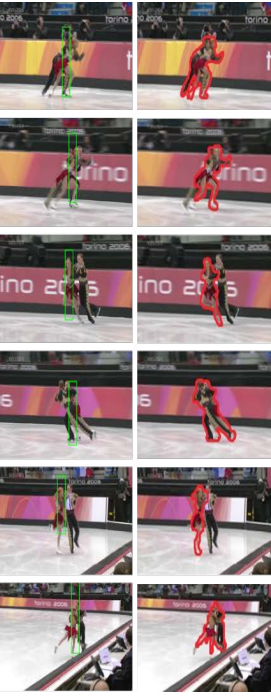

(e)

(e)
Figure 11. Skating video. (a) Original frame. (b) Cam-shift tracking. (c) Mean-shift tracking. (d) Kalman-filter tracking. (e) Particle-filter tracking. (f) Proposed method.

The experiment results for the Cam-shift algorithm and the Mean-shift algorithm show that the tracking of the regions of a single object is relatively accurate. The detection results of the tracking method for which the Kalman-filter algorithm is used are different depending on how the tracking area from the colors in the foreground and the background of the video frame are lost. The tracking method for which the Particle-filter algorithm was used shows a high-accuracy tracking result because it contains the color information of the object that makes it possible to retrace the target. The proposed method was performed for the achievement of an accurate tracking along the contour of the object, and it showed a high accuracy such as that of the Particle-filter algorithm; however, the third frame of Figure 8 shows that the target track was missed from the covering of the target by the other objects. And, when a similar color distribution is required across all of the objects, the proposed-method results show that its capability to track an independent object is more accurate than that of the conventional object-tracking methods.

The numbers of frames for the detection of only the area of the object in the image for an objective evaluation of the results were measured and compared for accuracy. When two or more similar objects display an obstruction or a configuration, the tracked number of the object area that is besides the target was classified as a false detection.

Table 1 is a comparison of the tracking accuracy of the proposed method and the conventional method table. The proposed method showed a higher accuracy than both the conventional Cam-shift algorithm and the Mean-shift algorithm. Also, compared to the experiment results of the Particle-filter algorithm, poor-accuracy results are shown for the proposed method according to the variety of the videos, but a high-performance experiment result for the tracking of a single object in a complex video frame is also shown.

\section{Conclusion}

In this paper, a method for accurate real-time object tracking in video is proposed. The proposed method was

Table 1. The number of exactly detected frames

\begin{tabular}{llllll}
\hline Video Type & Cam-shift & Mean-shift & $\begin{array}{l}\text { Kalman } \\
\text { filter }\end{array}$ & Particle filter & $\begin{array}{l}\text { Proposed } \\
\text { Method }\end{array}$ \\
\hline Ballet $(382$ & $67.80 \%(259$ & $75.13 \%(287$ & $9.42 \%(36$ & $81.67 \%(312$ & $89.26 \%(341$ \\
frames $)$ & frames $)$ & frames $)$ & frames $)$ & frames $)$ & frames $)$ \\
Car $(299$ & $0.66 \%(2$ & $71.23 \%(213$ & $75.91 \%(227$ & $93.31 \%(279$ & $91.63 \%(274$ \\
frames $)$ & frames $)$ & frames $)$ & frames $)$ & frames $)$ & frames $)$ \\
Bottles $(595$ & $23.02 \%(137$ & $27.89 \%(166$ & $79.49 \%(473$ & $94.78 \%(564$ & $92.10 \%(548$ \\
frames $)$ & frames $)$ & frames $)$ & frames $)$ & frames $)$ & frames $)$ \\
Peoples $(1904$ & $30.25 \%(576$ & $41.17 \%(784$ & $34.03 \%(648$ & $79.46 \%(1513$ & $85.34 \%(1625$ \\
frames) & frames $)$ & frames $)$ & frames $)$ & frames $)$ & frames $)$ \\
Skating $(706$ & $66.85 \%(472$ & $73.93 \%(522$ & $51.98 \%(367$ & $88.38 \%(624$ & $90.79 \%(641$ \\
frames $)$ & frames) & frames $)$ & frames $)$ & frames $)$ & frames $)$ \\
\hline
\end{tabular}


used for the HSV color space to achieve the accurate object tracking in a variety of imaging environments. Also, the use of the color information and the contour information for the proposed method to track the unique characteristics of the object resulted in an accuracy that is higher than those of the conventional methods. According to the experiment results, the tracking accuracy of the proposed method for a single object is $30 \%$ more than those of the Cam-shaft algorithm and the Mean-shift algorithm. In addition, the results show that, for a single object, the tracking for complex video frames is more accurate when the proposed method is used compared to that when the Particle-filter algorithm is used. The disadvantage of the proposed algorithm was also identified, however, whereby a relatively slow operation speed is due to the pursuit of accuracy. Further research is necessary, as the sole focus of the present study is the algorithm operation speed.

\section{Acknowledgements}

This study is sponsored by the 2016 research fund of Kwangwoon University.

\section{References}

1. Lee G. A modified grabcut using a clustering technique to reduce image noise, symmetry, 8.7:64GUO; 2016. p. 1-9.

2. Padmavathi S, Naveen CR, Kumari VA. Vision based vehicle counting for traffic congestion analysis during night time. Indian Journal of Science and Technology. 2016; 9(20):1-6.

3. Jazayeri A. Vehicle detection and tracking in car video based on motion model. IEEE Transactions on Intelligent Transportation Systems. 2011; 12(2):583-95.

4. Zakaria M, Shahrel A, Suandi. Malaysian car number plate detection system based on template matching and colour information. IJCSE. 2010; 2:1159-64.

5. Ning J. Robust mean-shift tracking with corrected background-weighted histogram. IET Computer Vision. 2012; 6(1):62-9.

6. Exne D. Fast and robust CAMShift tracking. IEEE Computer Society Conference on Computer Vision and Pattern Recognition-Workshops; 2010. p. 9-16.

7. Ganesan P. Satellite image segmentation based on $\mathrm{YCbCr}$ color space. Indian Journal of Science and Technology. 2015; 8(1):35-41.

8. Sabine S, Robert B, Steve S. Standard RGB color spaces. Color and Imageing Conference. Society for Imaging Science and Technology; 1999. p. 127-34.

9. Sural S, Qian G, Pramanik S. Segmentation and histogram generation using the HSV color space for image retrieval. 2002 IEEE International Conference on Image Processin; 2002. p. 589-92.

10. Dharavath K, Talukdar FA, Laskar RH. Improving face recognition rate with image preprocessing. Indian Journal of Science and Technology. 2014; 7(8):1170-5.

11. Allen JG, Xu RYD, Jin JS. Object tracking using camshift algorithm and multiple quantized feature spaces. Proceedings of the Pan-Sydney area workshop on Visual information processing. Australian Computer Society, Inc; 2004. p. 3-7.

12. Jung-ho L, Woong-hee L, Dong-seok J. Object tracking method using back-projection of multiple color histogram models. Proceedings of the 2003 International Symposium on Circuits and Systems, ISCAS’03; 2003. p. 668-71.

13. Branko R. Beyond the KalmanFilter: Particle filters for tracking applications. Boston: Artech house; 2004. p. 1-47.

14. Qi Z. Particle filter object tracking based on Harris-SIFT feature matching. Procedia Engineering. 2012; 29:924-9. 